(gonorrhea, chlamydia, syphilis). Using inverse probability of treatment weighted (IPTW) regression to account for preincarceration poverty, psychopathology, drug use, and STI risk, we estimated risk ratios (RRs) and 95\% confidence intervals (CIs) for associations between incarceration and outcomes and assessed differences by city.

Results Approximately 14\% had been incarcerated in the past six months. Controlling for site, incarceration predicted multiple partnerships (RR: 1.20, 95\% CI: 1.06-1.36) and incident STI (RR: 1.08, 95\% CI: 1.00-1.16). Associations with multiple partnerships and STI differed by city (joint test of interaction, $\mathrm{p}$ value $<0.05$ ). Incarceration was most strongly associated with multiple partnerships (RR: 1.69, 95\% CI: 1.38-2.04) and STI (RR: 1.31, 95\% CI: 1.04-1.64) in Washington DC. In other cities, STI RRs ranged from 0.95 to 1.08 and were not significant at the 0.05 level. Incarceration was associated with multiple partnerships in New York (RR: 1.25, 95\% CI: 1.01-1.55) and Boston (RR: 1.31, 95\% CI: 1.081.58), while RRs ranged from 0.87 to 1.08 and were not significant in other cities.

Conclusion Recent incarceration impacts STI risk among BMSM in Washington DC and the northeastern United States. Disclosure No significant relationships.

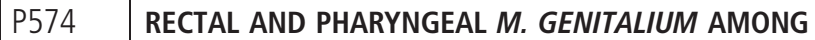 MEN WHO HAVE SEX WITH MEN (MSM): RESULTS FROM A LONGITUDINAL COHORT STUDY}

${ }^{1}$ Lindley Barbee*, ${ }^{2}$ Matthew Golden, ${ }^{3}$ Olusegun Soge, ${ }^{1}$ Micaela Haglund, ${ }^{4}$ Winnie Yeung, ${ }^{5}$ Christine Khosropour, ${ }^{6}$ James Hughes, ${ }^{5}$ Lisa Manhart. ${ }^{1}$ University of Washington, Medicine, Seattle, USA; ${ }^{2}$ University of Washington, Medicine and Epidemiology, Seattle, USA; ${ }^{3}$ University of Washington, Global Health and Medicine (Infectious Diseases), Seattle, USA; ${ }^{4}$ University of Washington, Medicine, Seattle WA, USA; ${ }^{5}$ University of Washington, Epidemiology, Seattle, USA; ${ }^{6}$ University of Washington, Biostatistics, Seattle, USA

\subsection{6/sextrans-2019-sti.648}

Background Little is known about the natural history of rectal and pharyngeal Mycoplasma genitalium (MG).

Methods From March 2016 to December 2018, we enrolled 140 high-risk MSM in a 12-month cohort study. Eligibility criteria included: age $\geq 18$ years, report of receptive anal intercourse, and $\geq 1$ of the following in $\leq 12$ months: (1) gonorrhea, chlamydia or syphilis; (2) methamphetamine or popper use, or (3) $\geq 2$ sex partners in $\leq 2$ months or $\geq 5$ in $\leq 12$ months. Each week, men collected pharyngeal and rectal swabs and completed an electronic sex diary. We tested swabs for MG (Aptima, Hologic, Inc.) at study completion. Infection was defined as $\geq 2$ consecutive positive tests; clearance was $\geq 2$ consecutively negative tests. We calculated person-weeks-atrisk to estimate incidence and used Kaplan Meier curves to estimate duration of infection. We censored subjects who had a positive swab in the final study week, received doxycycline or azithromycin and obtained clearance in the following 3 weeks, or were lost-to-follow-up. None received moxifloxacin. Results At week 1, the prevalence of rectal and pharyngeal MG was 8\% (4/48) and 2\% (1/48), respectively. Among 48 men with results from 3,579 specimens, 12 (25\%) had $\geq 1$ rectal, and $5(10 \%)$ had $\geq 1$ pharyngeal $M G$ infection. In 1,504 person-weeks-at-risk, rectal MG incidence was 38 (95\%
CI: 19-68) infections per 100 person-years. In 1,715 personweeks-at-risk, pharyngeal MG incidence was 15 (95\%CI: 5$35)$ per 100 person-years. Forty percent (6/15) of rectal and $50 \%(3 / 6)$ of pharyngeal infections were censored. Rectal and pharyngeal infections had estimated median durations of 30 (95\%CI: 6 - undefined) and 16 (95\% CI: 3 - undefined) weeks, respectively. Blips, single positive tests that did not meet criteria for infection, occurred in $8(17 \%)$ men with 17 rectal and $4(8 \%)$ men with 10 pharyngeal MG-positive tests.

Conclusion xtragenital, MG, including pharyngeal infection, is common and persistent among high-risk MSM.

Disclosure No significant relationships.

\section{P575 PREVALENCE AND RISK FACTORS OF ANAL HUMAN PAPILLOMAVIRUS (HPV) INFECTIONS AMONG MSM IN BALI, INDONESIA}

${ }^{1}$ Irvin Romyco*, ${ }^{1}$ Lucyan Umboh, ${ }^{2}$ Silvera Erari, ${ }^{3}$ Steve Wignall. ${ }^{1}$ FHI360-LINKAGES, Coptc (continuum of HIV Prevention, Treatment and Care), Jayapura, Indonesia; ${ }^{2}$ INA-RESPOND, Clinical Research Data Manager, Jakarta, Indonesia; ${ }^{3}$ Bali Peduli, Founder, Denpasar, Indonesia

\subsection{6/sextrans-2019-sti.649}

Background It is estimated that the prevalence of anal HPV infection is $15 \%, 60 \%$ and $95 \%$ among heterosexual men, MSM who are HIV negative and HIV positive MSM, respectively. Such high prevalence of anal HPV infection among MSM is related to 44 times higher incidence of anal cancer. We conducted a study to measure the prevalence and determine risk factors associated with anal HPV among MSM in Bali, Indonesia using a GeneXpert ${ }^{\circledR}$ platform.

Methods Between July 2017 and July 2018, 253 MSM were recruited in 2 MSM clinics in Bali, Indonesia. Sociodemographic and sexual behavioral characteristics were collected anonymously via self-completed questionnaire using tablet. The anal swab specimen was then collected, and HPV was tested using GeneXpert ${ }^{\circledR}$.

Results 174/242 (71.9\%) participants have positive results for any types of anal HPV, of which 95 (54.60\%) were among persons aged 20-29. The prevalence of high-risk HPV types (HPV 16 and HPV 18-45) was 109/242 (45\%), while the low-risk HPV was 155/242 (64\%). Age, relationship status, employment status, condom use, and sex position were factors associated with increased risks of contracting HPV, particularly high-risk HPV types. Young age between 20-29 accounted 5.05-fold (1.14-22.32, 95\% CI) risks acquiring HPV type 16 compared to other age group. Those who were open to a relationship have 6 times $(0.68-52.75,95 \% \mathrm{CI})$ higher risks of anal HPV 16 as opposed to those who were married. MSM who were employee increased almost 5 times (0.65-38.43, 95\% CI) risks of anal types of HPV 16.

Conclusion As the first study ever reported about the prevalence and risk factors of anal HPV infections among MSM in Indonesia, this study showed similar results and findings with other studies from other countries. Vaccination program can be considered to reduce the preponderant burden of anal HPV among MSM in Bali.

Disclosure No significant relationships. 